\title{
Family matters for coronavirus disease and vaccines
}

\author{
David J. Bean ${ }^{1}$ and Manish Sagar ${ }^{1,2}$ \\ 'Department of Microbiology, and '2Department of Medicine, Boston University School of Medicine, Boston, Massachusetts, USA.
}

\begin{abstract}
The increasing frequency of pathogenic coronaviruses in the human population has raised public health concerns about possible future pandemics. It is critical to understand whether immune responses to the current circulating coronaviruses provide protection against related viruses or those that may emerge in the future. In this issue of the $J \mathrm{Cl}$, Dangi, Palacio, and co-authors detail the extent of coronavirus cross-protection following both vaccination and natural infection and ultimately used murine models to highlight the mechanism behind this heterotypic immunity. This study provides insight into the possibility of a pan-coronavirus vaccine that could protect humans against future coronavirus outbreaks.
\end{abstract}

\section{Diverse coronaviruses}

The repeated emergence of different pathogenic human coronaviruses over the past 20 years has highlighted the impact of these viruses on public health and the need for a vaccine that can protect against future outbreaks. Animals, such as bats, harbor a wide variety of coronaviruses, and zoonotic spillovers are the likely source of both the current coronavirus infections and potentially of future viral outbreaks in humans (1). Currently, the known human coronaviruses cause a range of disease outcomes, from the four endemic coronaviruses (eCoVs) that are often the etiologic agent for the common cold, to the more pathogenic coronaviruses that can cause severe disease and even death for some individuals (2). Severe acute respiratory syndrome coronavirus 1 (SARS-CoV-1) emerged in 2003, and infections were associated with a mortality rate of approximately $10 \%$ (3). Fortunately, transmission chains faded fairly quickly as a result of effective containment measures. Middle East respiratory syndrome caused by a related coronavirus (MERS-CoV) infection appeared in 2012, resulting in severe pneumonia and mortality rates reaching nearly $40 \%$ (4). Transmission of MERS-CoV remains mostly geographically restricted, although cases still appear to this day. SARS-CoV-2, which causes coronavirus disease 2019 (COVID-19), quickly became a worldwide pandemic after March 2020, primarily because of the high transmissibility of the virus and the relatively late implementation of public health measures (5-7). The SARSCoV-2 disease course varies from asymptomatic to death and depends on a variety of factors including age and preexisting medical conditions. Despite the range in disease outcomes, these diverse coronaviruses have the same genotypic architecture, share conserved gene sequences, and often use similar proteins and cellular processes to infect and replicate in host cells $(8,9)$. Given these relationships, it has always been an intriguing possibility that a previous infection with one or more of the human coronaviruses may provide heterotypic immunity that protects against subsequent infection and ameliorates disease outcomes for the other members of this extended coronavirus family.

\section{Heterotypic immunity among coronaviruses}

Preexisting heterotypic immunity may provide a partial explanation for the

\section{Delated Article: https://doi.org/10.1172/JCl151969}

Conflict of interest: The authors have declared that no conflict of interest exists.

varying outcomes observed in SARSCoV-2-infected individuals, yet this concept remains highly controversial (10, 11). Some, but not all, investigations have demonstrated that individuals with prior eCoV infection may be protected against SARS-CoV-2 acquisition and may have substantially lower mortality rates and less severe COVID-19 after SARS-CoV-2 infection (12-14). These discrepant results are not necessarily unexpected or surprising and may be explained by a range of factors. Nearly all humans have been infected by one or more of the eCoVs sometime during their lifetime, eliciting humoral and cellular immune memory against the infecting coronavirus. However, immune memory against coronaviruses is generally believed to be relatively short-lived, and individuals are repeatedly infected with the various circulating eCoVs (15, 16). The magnitude, quality, and breadth of this immunological footprint against other coronavirus family members likely depends on numerous variables, such as recency, severity, frequency, and sequence similarity. Only examination of large cohort data and routine longitudinal sampling would be potentially adequately powered to account for these diverse factors that affect heterotypic immunity among the various human coronaviruses.

In this issue of the JCI, Dangi, Palacio, and colleagues examined human samples and used murine models to understand heterotypic immunity among coronaviruses (17). Similar to previous studies, they show that SARS-CoV-2-infected and -vaccinated individuals possessed cross-reactive antibodies against some other coronaviruses (18). Importantly, subsequent mouse studies by Dangi, Palacio, and co-authors overcame some of the highlighted confounding variables present in natural history studies in humans, such as the type, timing, severity, and number of previous coronavirus infections. In their mouse model, the researchers demonstrate that diverse SARS-CoV-1 or SARS-CoV-2 vaccines, similar to the ones being used and in development 


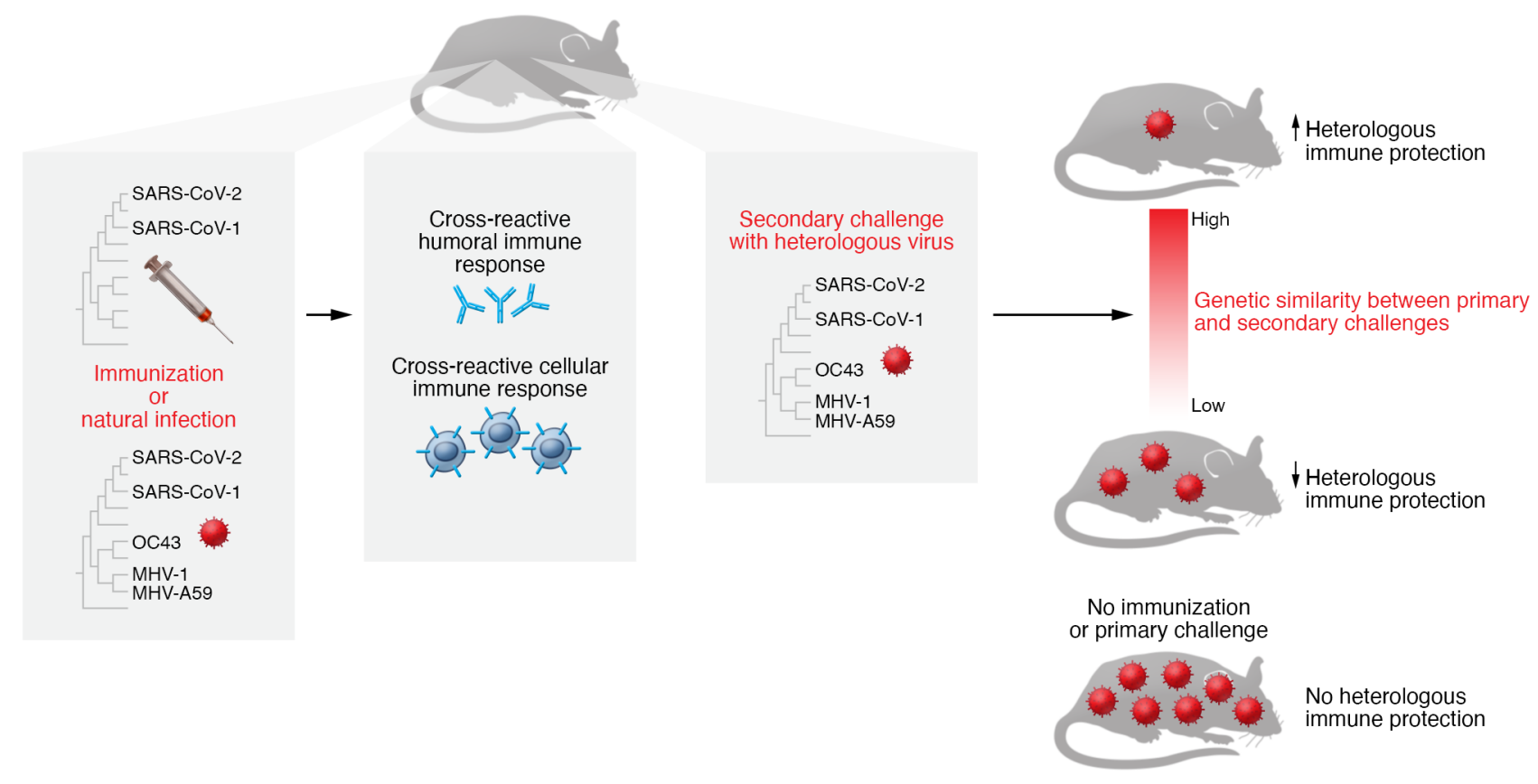

Figure 1. Coronavirus infection and vaccination induce antibodies and cellular responses that can provide heterotypic immunity against related coronaviruses. Mice vaccinated with various coronavirus vaccines produce antibodies and T cells that cross-react with related viruses, such as the common cold coronavirus OC43 or mouse hepatitis viruses MHV-1 and MHV-A59.

in the present day, elicited antibodies against a diverse range of coronaviruses (Figure 1). These immunizations provided protection against a challenge with a heterologous virus and lowered virus levels of the infecting coronavirus. In addition, mice infected and challenged with heterologous coronaviruses also showed heterotypic immune responses, similar to the results observed in the human vaccination studies. The authors further elucidated that the SARS-CoV-1 spike protein immunization induced both humoral and cellular responses against SARS-CoV-2. They validated the role of cross-reactive $\mathrm{T}$ cells and antibodies by showing that a $\mathrm{T}$ cell-based vaccine and passive infusion of antibodies generated from SARSCoV-2 vaccination or infection protected mice against disease after heterologous coronavirus challenge. Collectively, these comprehensive studies provide compelling evidence that coronavirus infection and vaccinations elicit heterotypic immunity against other virus family members. Importantly, the mouse studies provide the platform and framework for future studies aimed at examining the underlying protective mechanisms (17). For instance, future mouse studies can be utilized to understand the variable contribu- tion of peripheral blood versus lung-resident heterotypic immunity in protection against virus acquisition and disease manifestations.

\section{Combating the next coronavirus}

The results from Dangi, Palacio, and colleagues further suggest that viruses with greater sequence similarity elicit more potent cross-reactive immune responses and stronger cross-protection after virus challenge (Figure 1) (17). In some respects, these results confirm the observed interactions between SARS-CoV-1 and SARSCoV-2. SARS-CoV-1 and SARS-CoV-2 have a higher level of sequence similarity than that seen in the pairwise comparisons among the other human coronaviruses (6). Survivors of SARS-CoV-1 infection have some of the broadest and most potent SARS-CoV-2 antibodies after SARS-CoV-2 infection or vaccination (19, 20). This observation implies that SARSCoV-1 infection-elicited antibodies undergo further somatic hypermutation after encountering similar epitopes present in the SARS-CoV-2 spike protein. In addition, survivors of SARS-CoV-1 have detectable and higher levels of SARS-CoV-2-reactive T cells than do uninfected individuals (21).
Future human studies will need to investigate whether SARS-CoV-2 vaccination or infection protects against eCoVs to bolster this notion of heterotypic immunity. Additional experiments should examine the influence of recent $\mathrm{eCoV}$ infection on the immune response after a SARS-CoV-2 infection or vaccination, and whether it improves the breadth and potency of antibodies and increases cross-reactive $\mathrm{T}$ cell levels. These types of inquiries will have crucial importance for the efforts to develop vaccines that can protect against the current and future coronaviruses.

The greatest limitation in the mouse studies by Dangi, Palacio, and co-authors and the most important variable in the context of human infections is time. In the murine studies, immune responses were examined, or mice were challenged, within a few weeks of vaccination or infection (17). The durability and longterm potency of a heterotypic immune response remains uncertain. For coronaviruses, even homotypic immunity is relatively short, and humans are repeatedly infected with the different eCoVs because of waning immunity. This decline in immunity has also been observed with SARS-CoV-2, as reinfections after a prior infection and breakthrough infec- 
tions after vaccination are increasingly documented (22-24). The frequency of reinfections and breakthroughs increases with longer time spans from the prior infection or vaccination, and the heterotypic, as compared with these homotypic, immune responses are likely to be even less potent and durable. This waning immunity against coronaviruses presents a problem and suggests that repetitive infections or immunization on a periodic basis with different coronavirus family members may be required to boost and refresh immune responses. Even though SARS-CoV-2 repeat or breakthrough infections and eCoVs mostly cause mild disease, natural infections may be unsafe because of the variable clinical outcomes and the possible long-term side effects in healthy individuals (25). Serial vaccinations at defined intervals may offer the best solution to boost the immune response against both the vaccine immunogen and a heterologous coronavirus. This periodic immune refresher may not only help us battle the current circulating coronaviruses but also the next outbreak strain. As we begin to emerge into a post-pandemic world, we need to contemplate how to best utilize heterotypic immune strategies to combat the next coronavirus family member.

\section{Acknowledgments}

This work was supported by K24-AI145661 and P30-AI042853. DJB was supported by $\mathrm{NIH}$ grant T32-5T32AI00730928. The funders had no role in the decision to submit this article for publication.
Address correspondence to: Manish Sagar, Boston University, 650 Albany Street, Room 647, Boston, Massachusetts 02118, USA. Phone: 617.414.5239; Email: msagar@bu.edu.

1. Ye Z-W, et al. Zoonotic origins of human coronaviruses. Int J Biol Sci. 2020;16(10):1686-1697.

2. Paules CI, et al. Coronavirus infections-more than just the common cold. JAMA. 2020;323(8):707-708.

3. Leung GM, et al. The epidemiology of severe acute respiratory syndrome in the 2003 Hong Kong epidemic: an analysis of all 1755 patients. Ann Intern Med. 2004;141(9):662-673.

4. Assiri A, et al. Epidemiological, demographic, and clinical characteristics of 47 cases of Middle East respiratory syndrome coronavirus disease from Saudi Arabia: a descriptive study. Lancet Infect Dis. 2013;13(9):752-761.

5. Zhou P, et al. A pneumonia outbreak associated with a new coronavirus of probable bat origin. Nature. 2020;579(7798):270-273.

6. $\mathrm{Hu} \mathrm{B}$, et al. Characteristics of SARS-CoV-2 and COVID-19. Nat Rev Microbiol. 2021;19(3):141-154.

7. Sinha P, et al. Coronavirus disease 2019 mitigation strategies were associated with decreases in other respiratory virus infections. Open Forum Infect Dis. 2021;8(6):ofab105.

8. Bergmann CC, Silverman RH. COVID-19: coronavirus replication, pathogenesis, and therapeutic strategies. Cleve Clin JMed. 2020;87(6):321-327.

9. Sariol A, Perlman S. Lessons for COVID-19 immunity from other coronavirus infections. Immunity. 2020;53(2):248-263.

10. Anderson EM, et al. Seasonal human coronavirus antibodies are boosted upon SARS-CoV-2 infection but not associated with protection. Cell. 2021;184(7):1858-1864.

11. Poston D, et al. Absence of severe acute respiratory syndrome coronavirus 2 neutralizing activity in prepandemic Sera from individuals with recent seasonal coronavirus infection. Clin Infect Dis. 2021;73(5):e1208-e1211.

12. Sagar M, et al. Recent endemic coronavirus infection is associated with less-severe COVID-19. J Clin Invest. 2021;131(1):e143380.

13. Kaplonek P, et al. Early cross-coronavirus reactive signatures of humoral immunity against COVID-19. Sci Immunol. 2021;6(64):eabj2901.

14. Bartsch YC, et al. Humoral signatures of protective and pathological SARS-CoV-2 infection in children. Nat Med. 2021;27(3):454-462.

15. Callow KA, et al. The time course of the immune response to experimental coronavirus infection of man. Epidemiol Infect. 1990;105(2):435-446.

16. Edridge AWD, et al. Seasonal coronavirus protective immunity is short-lasting. Nat Med. 2020;26(11):1691-1693.

17. Dangi T, et al. Cross-protective immunity following coronavirus vaccination and coronavirus infection. J Clin Invest. 2021;131(24):e151969.

18. Hicks J, et al. Serologic cross-reactivity of SARSCoV-2 with endemic and seasonal betacoronaviruses. J Clin Immunol. 2021;41(5):906-913.

19. Shiakolas AR, et al. Cross-reactive coronavirus antibodies with diverse epitope specificities and Fc effector functions. Cell Rep Med 2021;2(6):100313.

20. Tan C-W, et al. Pan-sarbecovirus neutralizing antibodies in BNT162b2-immunized SARS-CoV-1 survivors. $N$ Engl J Med. 2021;385(15):1401-1406.

21. Le Bert N, et al. SARS-CoV-2-specific T cell immunity in cases of COVID-19 and SARS, and uninfected controls. Nature. 2020;584(7821):457-462.

22. Bean DJ, et al. SARS-CoV-2 reinfection associates with unstable housing and occurs in the presence of antibodies [published online November 10, 2021]. Clin Infect Dis. https://doi. org/10.1093/cid/ciab940.

23. Townsend JP, et al. The durability of immunity against reinfection by SARS-CoV-2: a comparative evolutionary study [published online October 1, 2021]. Lancet Microbe. https://doi. org/10.1016/S2666-5247(21)00219-6.

24. Bergwerk M, et al. Covid-19 breakthrough infections in vaccinated health care workers. $N$ Engl J Med. 2021;385(16):1474-1484.

25. Schmidt C. COVID-19 long haulers. Nat Biotechnol. 2021;39(8):908-913. 